\title{
A FIRST-PRINCIPLES THERMODYNAMIC APPROACH TO ORDERING IN Ni-Mo ALLOYS
}

\author{
A. ARYA ${ }^{1}$, S. BANERJEE ${ }^{1} \uparrow$, G. P. DAS ${ }^{2}$, I. DASGUPTA ${ }^{3}$, T. SAHA-DASGUPTA ${ }^{4}$ and \\ A. MOOKERJEE ${ }^{4}$
}

${ }^{1}$ Materials Science Division, Bhabha Atomic Research Centre, Mumbai 400 085, India, ${ }^{2}$ TP\&PE Division, Bhabha Atomic Research Centre, Mumbai 400 085, India, ${ }^{3}$ Department of Physics, Indian Institute of Technology, Powai, Mumbai 400 076, India and ${ }^{4}$ S.N. Bose National Centre for Basic Sciences, JDBlock, Sector III, Salt Lake, Calcutta 700 091, India

\begin{abstract}
There is a competition between several face centered cubic (FCC)-based ordered inter-metallic phases in Ni-Mo alloys containing 8-33 at\% Mo. The transformation behavior of these alloys in terms of ordering instabilities has been studied. First-principles tight-binding-linear muffin-tin orbital (TB-LMTO) method coupled with augmented space recursion (ASR) in conjunction with orbital peeling (OP) technique has been employed to extract the concentration dependent effective pair interactions. Further, the mean-field statistical mechanics based static concentration wave (SCW) model has been used to determine the free energies of these ordered phases as functions of temperature, composition and order parameter. This ASROP-SCW approach, applied to Ni-Mo alloy system, gives the correct ground state stability sequence as observed experimentally. Furthermore, it has been shown that such an approach can be used to study the complex transformation behavior involving several competing superstructures as well as competing first order and second order ordering processes.
\end{abstract}

Keywords: Order-disorder phenomena; Thermodynamics; Ni-Mo alloys; First principles calculations

\section{INTRODUCTION}

The Ni-rich side of the Ni-Mo system (see Fig. 1) [1] has three equilibrium ordered inter-metallic phases viz. $\beta-\mathrm{Ni}_{4} \mathrm{Mo}\left(\mathrm{D} 1_{\mathrm{a}}\right), \gamma-\mathrm{Ni}_{3} \mathrm{Mo}\left(\mathrm{DO}_{\mathrm{a}}\right)$ and $\delta-\mathrm{NiMo}$

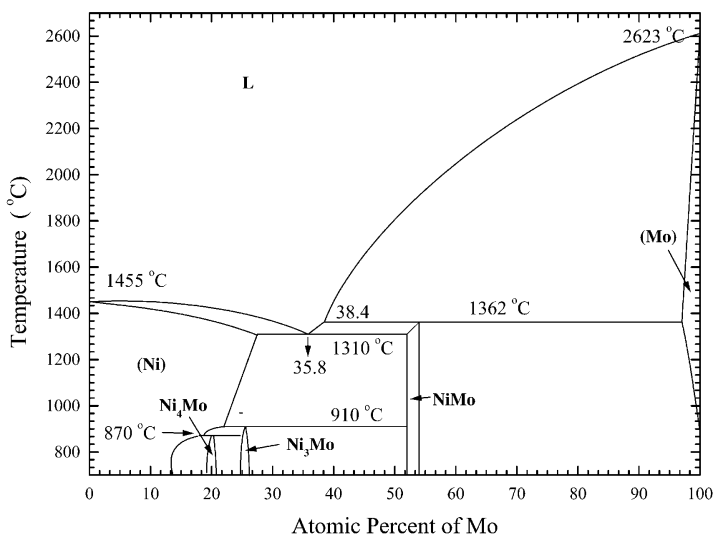

Fig. 1. The Ni-Mo phase diagram.
$\left(\mathrm{P} 2{ }_{1} 22_{1}\right)$. Some unique features of ordering transformation in this system have attracted the attention of several research groups. An extensive amount of research work [2-4] has been dedicated to understand the behavior of phase transformations that occur in this system $[5,6]$. There have been a number of studies on the ordering of $\mathrm{Ni}_{4} \mathrm{Mo}$ and $\mathrm{Ni}_{3} \mathrm{Mo}$ based alloys. It has been well established that these alloys, on quenching from the single phase FCC region, produce a short range ordered (SRO) state characterized by diffraction intensity at $\left\langle 1 \frac{1}{2} 0\right\rangle_{\mathrm{FCC}}$ positions and a complete extinction of intensity at $\langle 210\rangle_{\mathrm{FCC}}$ positions of the reciprocal space [7]. Such an SRO structure has been observed over a range of compositions (8-33 atomic \% Mo) in Ni-Mo alloys and in several other alloys like $\mathrm{Au}_{4} \mathrm{~V}, \mathrm{Au}_{4} \mathrm{Fe}, \mathrm{Au}_{4} \mathrm{Mn}, \mathrm{Au}_{3} \mathrm{Cr}$ [7, 8].

The development of the long range order (LRO) structures from the SRO state in the $\left\langle 1 \frac{1}{2} 0\right\rangle$ family of alloys has often been found to occur through a variety of intermediate stages $[4,5]$. In $\mathrm{Ni}_{4}$ Mo-based alloys, the initial stages of the SRO to LRO transition have been found to proceed in a continuous manner in a certain temperature range as evidenced by a continuous transfer of intensity from $\left\langle 1 \frac{1}{2} 0\right\rangle$ to $\frac{1}{5}\langle 420\rangle$ positions. At higher temperatures, however, the transition 
occurs by nucleation and growth of $\mathrm{D}_{\mathrm{a}}: \mathrm{Ni}_{4} \mathrm{Mo}$ particles. In $\mathrm{Ni}_{3} \mathrm{Mo}$-based alloys, the SRO to LRO transition has been observed to be more complex in that the SRO state initially decomposes into a phase mixture of two coherent ordered phases: (a) the metastable $\mathrm{Ni}_{2} \mathrm{Mo}\left(\mathrm{Pt}_{2} \mathrm{Mo}\right.$ type, Immm) and (b) the $\mathrm{Ni}_{4} \mathrm{Mo}$ $\left(\mathrm{D} 1_{\mathrm{a}}, \mathrm{B} 2 / \mathrm{m}\right)$. The stoichiometric $\mathrm{DO}_{22}$ structure has been found to form only under some special circumstances e.g. in ternary alloys where Mo is partially substituted by $\mathrm{Al}$ or Ta or in alloys aged at temperatures below $900 \mathrm{~K}$ for a long time.

The SRO structure in alloys exhibiting $\left\langle 1 \frac{1}{2} 0\right\rangle$ intensity maxima has been described by de Fontaine [9] in terms of concentration fluctuation with wave vectors close to $\left\langle 1 \frac{1}{2} 0\right\rangle$. Development of a $\left\langle 1 \frac{1}{2} 0\right\rangle$ concentration wave, in an FCC lattice, can produce two possible distinct real space structures depending upon the phase relationship of the concentration wave with FCC lattice. The first of these is a hypothetical "special point" structure proposed by Khachaturyan [10], which consists of an NNMM type stacking on a $\{420\}$ set of planes (also called $\mathrm{N}_{2} \mathrm{M}_{2}$ structure). A phase shift of this wave by $\pi / 4$ and a reduction in amplitude by a factor of $\sqrt{2}$ generate the so-called imperfectly ordered $\mathrm{DO}_{22}$ structure proposed by Okamoto and Thomas [11]. This consists of a sequence of Ni-rich (N), average (A) and Mo-rich (M) set of planes (called NAM structure). The $\mathrm{N}_{2} \mathrm{M}_{2}$ structure satisfies all the three Landau-Lifshitz symmetry rules, thereby making it a candidate for an order-disorder transition of second kind. The $\mathrm{N}_{2} \mathrm{M}_{2}$ structure can evolve by continuous ordering in the concentration range of $8-33 \%$ Mo. In the treatment of spinodal ordering [12], it has been shown that at temperatures below the $\left\langle 1 \frac{1}{2} 0\right\rangle$ ordering spinodal, the evolution of ordering can initiate by the amplification of an $\left\langle 1 \frac{1}{2} 0\right\rangle$ concentration wave.

Since no off-stoichiometric structure can be stable at $0 \mathrm{~K}$ (for the Nernst theorem requires that the entropy of a structure vanish at $0 \mathrm{~K}$ ), the $\mathrm{N}_{2} \mathrm{M}_{2}$ structure can not satisfy this requirement for $\mathrm{Ni}_{3} \mathrm{Mo}$ alloys. In such a situation, the alloy is expected to undergo a secondary ordering giving rise to a stoichiometric ordered structure or separate into a phase mixture of ordered structures. Kulkarni and Banerjee [4] considered three different cases of secondary ordering involving three different FCC special point concentration waves in the off-stoichiometric $\mathrm{N}_{2} \mathrm{M}_{2}$ structure generated by $\left\langle 1 \frac{1}{2} 0\right\rangle$ spinodal ordering: (a) generation of long wavelength concentration waves with k-vectors close to $\langle 000\rangle$ in an off-stoichiometric partially $\left\langle 1 \frac{1}{2} 0\right\rangle$ ordered alloy results in a phase separation of the alloy into a disordered solid solution and a nearly stoichiometric ordered $\mathrm{N}_{2} \mathrm{M}_{2}$ phase, (b) introduction of a $\langle 100\rangle$ concentration wave in a $\left\langle 1 \frac{1}{2} 0\right\rangle$ ordered alloy of $\mathrm{Ni}_{3} \mathrm{Mo}$ composition, gives rise to the $\mathrm{DO}_{22}$ structure (secondary ordering), and (c) secondary $\left\langle 1 \frac{1}{2} 0\right\rangle$ ordering in a direction perpendicular to the original ordering k-vector results in the formation of a " $\mathrm{N}_{3} \mathrm{M}$ " structure which contain an ordered arrangement of $\mathrm{D} 1_{\mathrm{a}}$ and $\mathrm{Pt}_{2} \mathrm{Mo}$ subunit cell clusters (Fig. 2).

The general tendency of the $\mathrm{Ni}_{3} \mathrm{Mo}$ alloy and some of its ternary derivatives to decompose into a mixture of the $\mathrm{Ni}_{2} \mathrm{Mo}$ and the $\mathrm{Ni}_{4} \mathrm{Mo}$ phases (avoiding the $\mathrm{DO}_{22}$ structure which satisfies the stoichiometry) has been rationalized in terms of nearly equal stabilities associated with the competing structures. The ground state analysis, reported by Kulkarni and Banerjee [4], pointed to the fact that in a $\left\langle 1 \frac{1}{2} 0\right\rangle$ alloy of $\mathrm{Ni}_{3}$ Mo stoichiometry, the super-imposition of either (i) an $\left\langle 1 \frac{1}{2} 0\right\rangle$ and an $\langle 100\rangle$ concentration waves or (ii) two mutually perpendicular $\left\langle 1 \frac{1}{2} 0\right\rangle$ concentration waves always results in a structure of lower internal energy than the off-stoichiometric structure generated by a single $\left\langle 1 \frac{1}{2} 0\right\rangle$ concentration wave. Moreover, the internal energy of an equimolar mixture of the $\mathrm{Ni}_{2} \mathrm{Mo}$ and the $\mathrm{Ni}_{4} \mathrm{Mo}$ phases is found to be same as that of the $\mathrm{Ni}_{3} \mathrm{Mo}$. The calculation of formation energies, used for such comparisons, are, however, based on suitable ratios of interaction energies for the first, the second and the third nearest neighbor pairs. More rigorous first-principles calculations have not been attempted so far.

Earlier [13], we had calculated volume-dependent effective pair interactions (EPIs) up to the second nearest-neighbor pairs using the TB-LMTO method in conjunction with Connolly-Williams inversion method (IM) in the octahedral-tetrahedral clusters

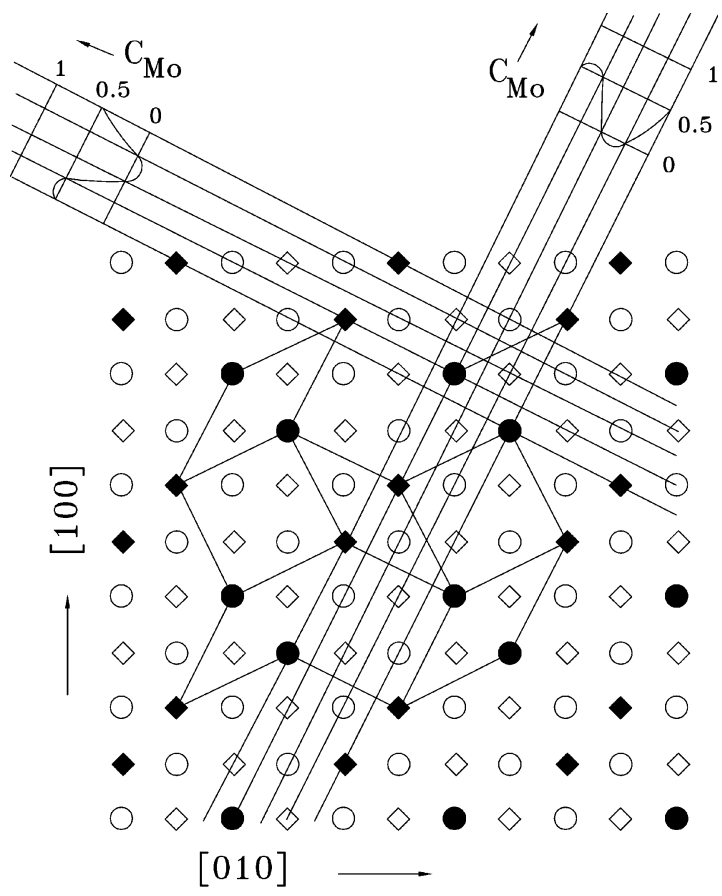

Fig. 2. The [001] projection showing atomic arrangement in the " $\mathrm{N}_{3} \mathrm{M}$ structure" generated by the superimposition of two perpendicular $\langle 420\rangle$ concentration waves. This structure comprises an arrangement of two variants, each of subunit cell clusters corresponding to the $\mathrm{N}_{4} \mathrm{M}$ (square-shaped) and the $\mathrm{N}_{2} \mathrm{M}$ (diamond-shaped) structures (Kulkarni et al. [4]). 
approximation. The basic idea of IM is to parameterize the cohesive energy of a given superstructure in terms of EPIs. In view of the fact that the stable/meta-stable ordered intermetallic phases in the Ni-Mo system are stabilized by considering up to the third or the fourth nearest-neighbor pair interactions, one needs to go to some higher (more complex) cluster approximations in the IM. This may require cohesive energy calculations of some meta-stable/unstable superstructures. On the other hand, the starting point of the ASR-OP method employed here is the completely disordered phase. We have therefore employed the ASR-OP method to generate energy and concentration dependent EPIs for the ground state instability analysis.

Further, we have used these EPIs in the mean-field statistical mechanics based static concentration wave (SCW) model to calculate the free energies of these ordered phases as a function of temperature, composition and order parameter. This method has been used earlier for studying the stability/meta-stability with respect to ordering and/or clustering reactions in the PdRh system [14]. The maximum point of the computed spinodal curve matched reasonably well (within $15 \%$ i.e. the error-bar of the mean field treatment) with the experimental maximum temperature of the miscibility gap in this system. Since the competing super-lattice structures $\left(\mathrm{N}_{4} \mathrm{M}, \mathrm{N}_{3} \mathrm{M}, \mathrm{N}_{2} \mathrm{M}\right.$, $\mathrm{N}_{2} \mathrm{M}_{2}$ ) in Ni-Mo system are all formed when specific concentration wave perturbations destabilize the perfectly disordered FCC alloy, it is appropriate to deploy the SCW approach in conjunction with the ASR-OP technique to investigate the ordering/disordering in this system.

The rest of the paper is organised as follows: in Section 2, we give the structural description of all the stable/metastable superstructures in terms of concentration waves. Section 3 is devoted to the computational details of the ASR-OP method. In Section 4 we present our results on the calculation of EPIs within ASR-OP method, and the results of groundstate as well as finite temperature phase stability within the SCW model. Finally the conclusions are given in Section 5.

\section{THE STRUCTURAL DESCRIPTION}

The coherent LRO structures which are encountered in the Ni-Mo alloy system belong to the $\left\langle 1 \frac{1}{2} 0\right\rangle$ family. These structures viz. $\mathrm{Ni}_{4} \mathrm{Mo}\left(\mathrm{D} 1_{\mathrm{a}}\right), \mathrm{Ni}_{3} \mathrm{Mo}$ $\left(\mathrm{DO}_{22}\right), \mathrm{Ni}_{2} \mathrm{Mo}\left(\mathrm{Pt}_{2} \mathrm{Mo}\right.$ type) and $\mathrm{Ni}_{2} \mathrm{Mo}_{2}$ (I4 $\left.{ }_{1} / \mathrm{amd}\right)$ can be described $[4,9,10]$ in terms of stacking of (420) planes (see Fig. 3) that contain either all Ni or all Mo atoms. These members of the (420) series are generally designated as $\mathrm{N}_{4} \mathrm{M}, \mathrm{N}_{3} \mathrm{M}, \mathrm{N}_{2} \mathrm{M}$ and $\mathrm{N}_{2} \mathrm{M}_{2}$, respectively.

The concentration wave description [10] of these superstructures is described by the concentration delta function, $Q(p)$, written as a function of the magnitude of the vector $\mathbf{p}$ in terms of the plane indices $\mathbf{p}=p\langle l m n\rangle . G(p)$ is equal to unity at the plane $p=0$ and zero elsewhere $(p=1, \ldots, N-1)$, so that the concentration Fourier spectrum along a specific vector $\mathbf{k}$ is given by

$$
G(k)=\frac{1}{N} \sum_{p=0}^{N-1} G(p) \exp [-2 \pi \text {.k.p.p }]
$$

For each structure, there are exactly $N(=2,3,4,5)$ Fourier waves of the same amplitude.

\section{1. $\mathrm{Ni}_{4} \mathrm{Mo}\left(\mathrm{D} 1_{a}\right)$ structure}

The unit cell of $\mathrm{Ni}_{4} \mathrm{Mo}$ structure along with its stacking description is shown in Fig. 2. It can be seen that the structure can be described as a layered structure of (420) layers with every fifth layer being occupied entirely by Mo atoms while the intervening four layers being filled by $\mathrm{Ni}$ atoms. The wave representation of such a structure or the occupation probability of Mo atoms on the $p$ th (420) layer can be expressed as (see Fig. 3)

$$
G(p)=c+\frac{2}{5} \eta\left(\cos \frac{2 \pi p}{5}+\cos \frac{4 \pi p}{5}\right)
$$

For stoichiometric composition, $\left(c_{\mathrm{Mo}} \equiv\right) c=\frac{1}{5}$, and for order parameter, $\eta=1$ (i.e. fully ordered), the (420) layered structure of $\mathrm{Ni}_{4} \mathrm{Mo}$ will, therefore, be, "Mo Ni Ni Ni Ni Mo...". This structure can be viewed as a super-imposition of $\frac{i}{5}\langle 420\rangle(i=1$ to 4$)$ waves.

\section{2. $\mathrm{Ni}_{3} \mathrm{Mo}\left(\mathrm{DO}_{22}\right)$ structure}

The $\mathrm{DO}_{22}$ structure can also be described as a (420) layered structure as shown in Fig. 2. The concentration wave description is given by (Fig. 3)

$$
G(p)=c+\frac{1}{4} \eta\left(2 \cos \frac{\pi p}{2}+\cos \pi p\right)
$$

The $\mathrm{DO}_{22}$ structure is the equilibrium structure of $\mathrm{Ni}_{3} \mathrm{~V}$ and also of some ternary alloys based on $\mathrm{Ni}_{3} \mathrm{Mo}$ where Mo is partially substituted by $\mathrm{Al}, \mathrm{Ti}$, Ta and $\mathrm{Nb}$. In a stoichiometric $(c=1 / 4)$ and fully ordered alloy $(\eta=1)$ the $(420)$ layering sequence is, "Mo Ni $\mathrm{Ni} \mathrm{Ni} \mathrm{Mo..."}$. It can be seen that apart from the four layer stacking of (420) planes, there exists a concentration modulation in the [010] direction in the $\mathrm{DO}_{22}$ structure. In fact, this structure can be viewed as a superposition of $\frac{\mathrm{i}}{4}\langle 420\rangle(i=1,3)$ and $\frac{2}{4}\langle 420\rangle \equiv\langle 100\rangle$ concentration waves.

\section{3. $\mathrm{Ni}_{2} \mathrm{Mo}$ ( $\mathrm{Pt}_{2} \mathrm{Mo}$ type) structure}

The $\mathrm{N}_{2} \mathrm{M}$ structure can be represented by a layered structure of (420) planes and the occupation probability of $\mathrm{M}$ atoms on the $p$ th plane can be described by the concentration wave (Figs 2 and 3 ) 

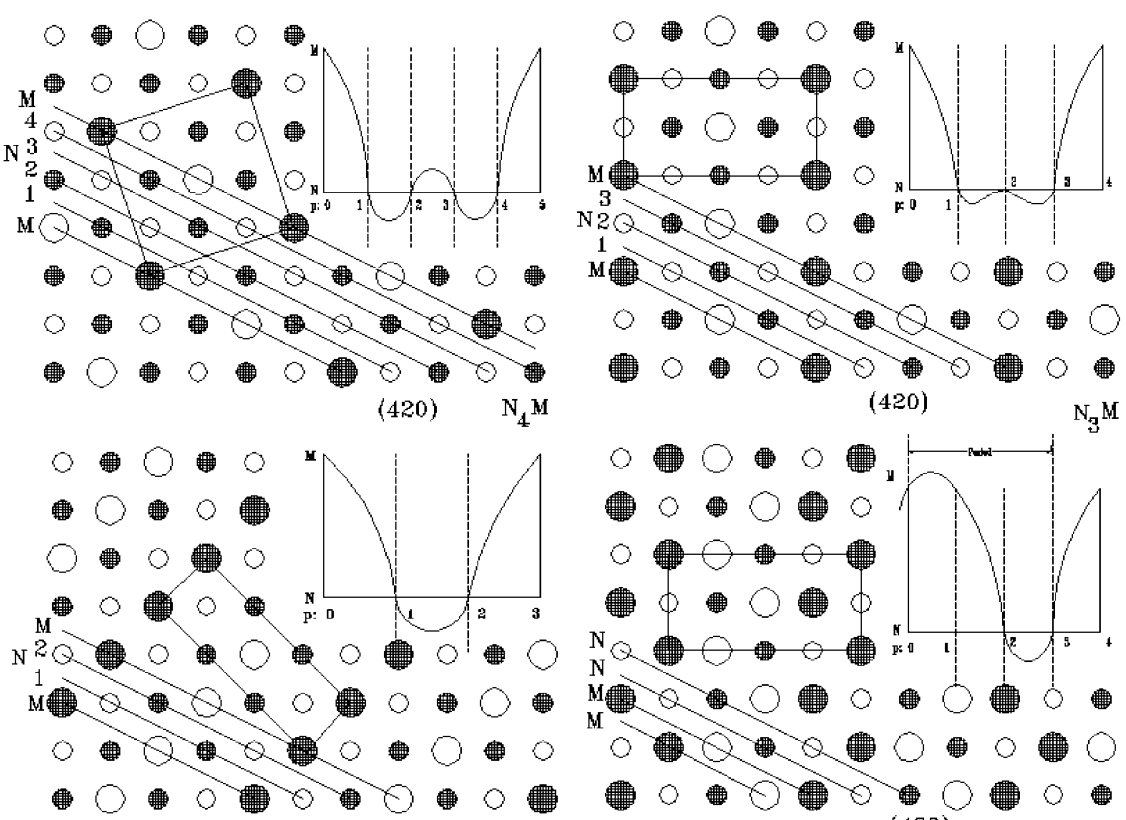

(420)

$\mathrm{N}_{2} \mathrm{M}$

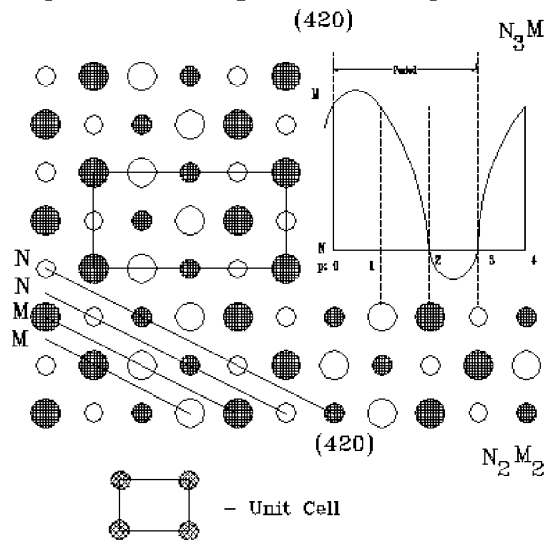

Fig. 3. The description of four FCC-based superstructures in terms of stacking of (420) planes in the [001] projections and static concentration waves. The sequences of $\mathrm{Ni}(\mathrm{N})$ and $\mathrm{Mo}(\mathrm{M})$ of (420) planes and subunit cell clusters are also shown.

$$
G(p)=c+\frac{2}{3} \eta\left(\cos \frac{2 \pi p}{3}\right)
$$

The stoichiometric fully ordered alloy exhibits a layering sequence of (420) planes of " Mo Ni Ni Mo...". This structure can be viewed as a super-imposition of $\frac{\mathrm{i}}{3}\langle 420\rangle(i=1,2)$ waves.

\section{4. $\mathrm{Ni}_{2} \mathrm{Mo}_{2}$ structure}

This structure can be constructed from the concentration wave with the $\left\langle 1 \frac{1}{2} 0\right\rangle$ wave vector. The significance of this vector is that it terminates on a special point in the FCC reciprocal lattice where symmetry elements intersect. The concentration wave associated with this structure is given by

$$
G(p)=c+\frac{\eta}{\sqrt{2}} \sin \frac{\pi}{2}\left(p+\frac{1}{2}\right)
$$

For $c=0.5$ and $\eta=1$, the layering sequence of (420) planes is, "Ni Ni Mo Mo Ni Ni...". This structure can be viewed as a super-imposition of $\frac{\mathrm{i}}{4}\langle 420\rangle(i=1,3)$ concentration waves.

\section{COMPUTATIONAL DETAILS}

3.1. The tight-binding augmented space recursion method

The recursion method [15: p. 216, 16] offers an alternative to the band structure method. This method is suitable for computing the local properties which are related to the diagonal elements of the resolvent, where it offers a large computational advantage over the band structure methods. Moreover, the recursion method is based on real space and so it does not require lattice periodicity for its operation, in contrast to the band structure methods. As a result, both ordered, disordered and systems with broken translational symmetry (such as surfaces) can be treated within the recursion method.

Within the TB-LMTO formalism the Hamiltonian is usually sparse. In the recursion method one exploits this sparseness and performs a unitary transformation on the local orbital basis, through a three term recurrence, to produce a tridiagonal representation of the Hamiltonian. The first orbital in the new basis is chosen to be the one on which the projected density of states is sought.

The augmented space formalism, introduced by Mookerjee [17] is a novel and conceptually attractive method for the calculation of the configuration averaged Green function of a disordered material. In this method one transforms the Hamiltonian describing a given disordered system to an effective non-disordered Hamiltonian, whose appropriate Green function matrix elements correspond to the configurational averages of the Green function of the original disordered system. This effective Hamiltonian operates on an enlarged Hilbert Space: which is the space $\mathscr{H}$ spanned by the TB-LMTO basis augmented by the space $\Phi$ spanned by all possible random configur- 
ations of the system. Configuration averages in real space are mapped onto particular matrix elements in this augmented space $\mathscr{H} \otimes \Phi$. We shall describe the main points of the formalism here and refer the readers to the original citation for details.

The disordered Hamiltonian is expressed as a function of a set of random variables: $\mathrm{H}\left(\left\{n_{\mathrm{i}}\right\}\right)$ whose probability densities are written as the spectral density of operators $\left\{M_{\mathrm{i}}\right\}$

$$
p\left(n_{\mathrm{i}}\right)=-\frac{1}{\pi} \mathscr{T} m\left\langle\gamma_{0}\left|\left(n_{\mathrm{i}} I-M_{\mathrm{i}}\right)^{-1}\right| \gamma_{0}\right\rangle
$$

where $\left|\gamma_{0}^{j}\right\rangle$ is the configuration ground state at the $i$ th site.

The augmented space theorem then states that:

$$
{ } G_{\mathrm{ii}} »=\left\langle i \otimes \gamma_{0}^{1} \otimes \gamma_{0}^{2} \ldots\left|\tilde{G}\left(\left\{M_{\mathrm{i}}\right\}\right)\right| i \otimes \gamma_{0}^{1} \otimes \gamma_{0}^{2} \ldots\right\rangle
$$

For a binary distribution of $n_{\mathrm{i}}$ with probability $c$ to be 1 and $(1-c)$ to be 0 :

$$
p\left(n_{\mathrm{i}}\right)=c \delta\left(n_{\mathrm{i}}-1\right)+(1-c) \delta\left(n_{\mathrm{i}}\right)
$$

the representation of $M_{\mathrm{i}}$ is a matrix of rank two:

$$
M^{(i)}=\left(\begin{array}{cc}
c & \sqrt{c(1-c)} \\
\sqrt{c(1-c)} & 1-c
\end{array}\right)
$$

The relation is exact and approximations are carried out in the exact numerical evaluation of $\left\langle G_{\mathrm{ii}}{ }\right\rangle$. Direct recursion in the augmented space has the advantage that it does not involve any single site mean-field approximation or the solution of self-consistent equations to determine the effective medium. The approximation required is in the termination of the continued fraction expansion of the configuration averaged Green function. Excellent termination procedures are available which retain most of the analytic properties of the exact Green function [18]. This method can treat both diagonal as well as off-diagonal disorder on an equal footing and preserves all the essential features of the recursive solution of the Schrödinger equation. The work-load of the recursion method is proportional to the size of the system. It can be further reduced if one exploits the symmetry of the Hamiltonian both in the real space as well as in the augmented space arising as a result of homogeneity of disorder. The rank of the irreducible subspace of the augmented space, on which the recursion is effectively carried out, is drastically reduced [19].

The starting point for the augmented space recursion is the most localized sparse tight-binding
Hamiltonian derived systematically from the LMTOASA theory $[20,21]$ and generalized to substitutionally disordered random binary alloys:

$$
\begin{array}{cc}
H_{\mathrm{RL}, \mathrm{R}^{\prime} \mathrm{L}^{\prime}}^{\beta} & =\hat{C}_{\mathrm{RL}} \delta_{\mathrm{RR}} \delta_{\mathrm{L} L^{\prime}}+\hat{\Delta}_{\mathrm{RL}} S_{\mathrm{RL}, \mathrm{R}^{\prime} L^{\prime}}^{\beta} \hat{\Delta}_{\mathrm{R}^{\prime} \mathrm{L}^{\prime}} \\
\hat{C}_{\mathrm{RL}} & =C_{\mathrm{RL}}^{A} n_{\mathrm{R}}+C_{\mathrm{RL}}^{B}\left(1-n_{\mathrm{R}}\right) \\
\hat{\Delta}_{\mathrm{RL}} & =\left(\Delta_{\mathrm{RL}}^{A}\right)^{1 / 2} n_{\mathrm{R}}+\left(\Delta_{\mathrm{RL}}^{B}\right)^{1 / 2}\left(1-n_{\mathrm{R}}\right)
\end{array}
$$

Here $R$ denotes the lattice sites and $L=(/ \mathrm{m})$ are the orbital indices (for transition metal $\leq 2$ ) $C_{\mathrm{RL}}^{A}, C_{\mathrm{RL}}^{B}$ and $\left(\Delta_{\mathrm{RL}}^{A}\right)^{1 / 2},\left(\Delta_{\mathrm{RL}}^{B}\right)^{1 / 2}$ are the potential parameters of the constituents $A$ and $B$ (in the most tight-binding representation of $\alpha=\beta$ ) of the alloy. $n_{\mathrm{R}}$ are the local site occupation variables which randomly take values 1 and 0 according to whether the site is occupied by an $A$ atom or not. From the discussion given above, it is clear that the representation of the Hamiltonian in the augmented space $\tilde{H}$ consists of replacing the local site occupation variables $\left\{n_{\mathrm{R}}\right\}$ by $\left\{\tilde{M}^{(R)}\right\}$, and is given by:

$$
\begin{aligned}
& \tilde{H}=\sum_{R L}\left(C_{\mathrm{RL}}^{B} \tilde{I}+\delta C_{\mathrm{RL}} \tilde{M}^{(R)}\right) \otimes \mathscr{P}_{R}+\ldots \\
& +\sum_{R L} \sum_{R^{\prime} L^{\prime}}\left(\left(\Delta_{\mathrm{RL}}^{B}\right)^{1 / 2} \tilde{I}+\delta \Delta_{\mathrm{RL}} \tilde{M}^{(R)}\right) S_{\mathrm{RL}, \mathrm{R}^{\prime} \mathrm{L}^{\prime}}^{\beta} \cdots \\
& \left(\left(\Delta_{\mathrm{R}^{\prime} \mathrm{L}^{\prime}}^{B}\right)^{1 / 2} \tilde{I}+\delta \Delta_{\mathrm{R}^{\prime} \mathrm{L}^{\prime}} \tilde{M}^{\left(R^{\prime}\right)}\right) \ldots \otimes \mathscr{T}_{\mathrm{RR}^{\prime}}
\end{aligned}
$$

where, $\delta C_{\mathrm{RL}}=\left[C_{\mathrm{RL}}^{A}-C_{\mathrm{RL}}^{B}\right] ; \quad \delta \Delta_{\mathrm{RL}}=\left[\left(\Delta_{\mathrm{RL}}^{A}\right)^{1 / 2}-\right.$ $\left.\left(\Delta_{\mathrm{RL}}^{B}\right)^{1 / 2}\right] \tilde{I}$ is the identity operator, $\mathscr{Q}_{\mathrm{R}}$ is the projection operator on the basis labeled by $R$ and $\mathscr{T}_{\mathrm{RR}^{\prime}}$ is the transfer operator $\left|R^{\prime}\right\rangle\langle R|$, other parameters have their usual meaning.

The TB-LMTO-ASR technique has been applied to a series of alloy systems with considerable success earlier [19]. These alloys involved among other things short-ranged ordering, clustering and local lattice distortions.

\subsection{Orbital peeling method}

The study of phase stability, starting from the disordered phase generally begins with setting up of a concentration fluctuation in a completely disordered medium and expansion of the change in configurational energy in terms of effective multi-site interactions. The dominant role is played by the EPI's which are defined to be

$$
J_{2} \equiv E_{\mathrm{ij}}=V_{\mathrm{AA}}+V_{\mathrm{BB}}-V_{\mathrm{AB}}-V_{\mathrm{BA}}
$$

which give the interchange energy associated with two sites embedded in an otherwise average medium, being occupied by A or B type of atom. These effective pair interactions can be expressed in terms of band structure contributions as 


$$
\begin{aligned}
E_{\mathrm{ij}} & =\int_{-\infty}^{E_{F}^{A A}} E \rho_{\mathrm{AA}}(E) d E+\int_{-\infty}^{E_{F}^{B B}} E \rho_{\mathrm{BB}}(E) d E \\
& -\int_{-\infty}^{E_{F}^{A B}} E \rho_{\mathrm{AB}}(E) d E-\int_{-\infty}^{E_{F}^{B A}} E \rho_{\mathrm{BA}}(E) d E
\end{aligned}
$$

where $E_{F}^{I J}$ and $\rho_{\mathrm{IJ}}(E)$ denote the Fermi energy and electronic density of states for a specific case where at the sites $i$ and $j$ in the random media are occupied by atoms of the type $(I, J=A, B)$. These EPIs can further be expressed in terms of the generalized phase shift $\delta(E)$ as

$$
E_{\mathrm{ij}}=\int_{-\infty}^{E_{F}} \mathscr{T} m \delta(E) d E
$$

with

$$
\delta(E)=\frac{1}{\pi} \log \operatorname{det} \frac{\ll G^{A A} » \ll G^{B B} »}{\ll G^{A B} » \ll G^{B A} »}
$$

where $« G^{I J}$ »'s are the configuration averaged Green functions with two atoms in all possible combinations embedded at sites $i$ and $j$.

The brute force method of calculating the EPIs would be to calculate the various averaged energy terms and then take the difference as in equation (14). However, determination of small differences of very large numbers often lead to numerical instabilities. A way out is to obtain the difference directly using the orbital peeling method [22]. The basic philosophy lies in removing the embedded atoms orbital by orbital and carrying out the recursion at each step.

According to Burke

$$
\frac{d}{d E} \log G=\sum_{\gamma-1}^{p-1} \frac{1}{E-Z_{\gamma}}-\sum_{\gamma-1}^{p} \frac{1}{E-P_{\gamma}}
$$

where $Z_{\gamma}\left(P_{\gamma}\right)$ 's are the zeros (poles) of the Green function expressed as continued fraction expansion evaluated up to $p$ levels. These can be obtained immediately from the continued fraction coefficients. Thus, the determination of effective pair interaction reduces to determination of zeroes and poles of peeled off Green functions with pair $\{I J\}$ embedded in an averaged medium. One employs the augmented space recursion coupled with the tight binding linearized muffin tin orbital method (TB-LMTO-ASR) for a first principle determination of configuration averaged peeled-off Green functions.

The orbital peeling combined with TB-LMTO-
ASR has been earlier applied to study the phase stability of binary alloy systems [23].

\section{RESULTS AND DISCUSSION}

\subsection{The EPIs}

We calculate the configurationally-averaged concentration-dependent effective pair interactions $J_{2}^{(m)}(c)$, up to the fourth (m) nearest-neighbor (NN) pairs for the Ni-Mo alloys using the first-principles ASR-OP method described in Section 3. We use the most-localized (tight-binding) set of potential parameters from self-consistent TB-LMTO calculations. A four-shell augmented space map (generated from a cluster of 400 sites) has been used, with interactions up to the first NN pairs, for these close-packed FCCbased structures.

The number of recursion steps has been restricted to eight and terminated with the Luchini-Nex terminator [18]. These EPIs in the $m$ th coordination sphere are defined as

$$
J_{2}^{(m)}=V_{\mathrm{AA}}^{(m)}+V_{\mathrm{BB}}^{(m)}-V_{\mathrm{AB}}^{(m)}-V_{\mathrm{BA}}^{(m)}
$$

which gives the interchange energy associated with two sites occupied by either A or B atom, embedded in an otherwise perfectly disordered averaged medium. Table 1 gives the calculated values of the EPIs up to the fourth NN shell for Ni-Mo alloys at $20,25,33$ and 50 at\% of Mo. The interactions are seen to be well-converged as a function of shell numbers. Figure 4 shows the variation of these EPIs as a function of concentration.

\subsection{SCW analysis}

In the SCW model $[10,24]$, the occupation probability $\mathcal{N}(\mathbf{r})$, at any lattice position, $\mathbf{r}(\mathrm{p})$, is given by the Fourier expansion as:

$$
\mathcal{N}(\mathbf{r})=\sum_{h=1}^{N} \mathcal{L}(\mathbf{k}) e^{-i \mathbf{k}(h) \cdot \mathbf{r}(p)}
$$

where $2(\mathbf{k})$ 's are the Fourier coefficients given by

Table 1. The concentration dependent EPIs, $J_{2}^{(m)}$, upto the fourth nearest neighbour pair $\left(m_{\max }=4\right)$ calculated using the ASR-OP method for the Ni-Mo alloys (in mRy)

\begin{tabular}{lccrr}
\hline & \multicolumn{4}{c}{ Composition (at\% Mo) } \\
\cline { 2 - 5 } & 20 & 25 & 33 & \multicolumn{1}{c}{50} \\
\hline$E_{\mathrm{F}}(\mathrm{mRy})$ & & & & \\
$J_{2}^{(1)}$ & 3.692 & 2.627 & 0.8492 & \multicolumn{1}{c}{0.5360} \\
$J_{2}^{(2)}$ & 0.8125 & 0.3074 & 0.4032 & -0.3291 \\
$J_{2}^{(3)}$ & -0.2647 & -0.2216 & -0.1636 & -0.1240 \\
$J_{2}^{(4)}$ & 0.0116 & 0.0207 & 0.0266 & 0.0261 \\
$J_{2}^{(2)} / J_{2}^{(1)}$ & 0.220 & 0.117 & 0.475 & 0.0261 \\
$J_{2}^{(3)} / J_{2}^{(1)}$ & -0.072 & -0.084 & -0.193 & -0.231 \\
\hline
\end{tabular}




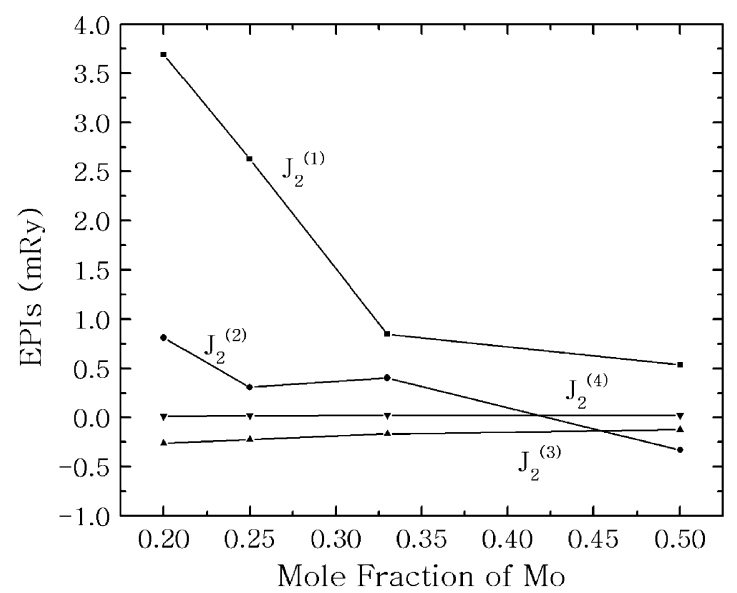

Fig. 4. The EPIs upto the fourth nearest-neighbor pairs, calculated using the ASR-OP technique, plotted as a function of Mo concentration.

$$
\mathscr{L}(\mathbf{k})=\frac{1}{N} \sum_{p=1}^{N} \mathcal{N}(\mathbf{r}) e^{i \mathbf{k}(h) \cdot \mathbf{r}(p)}
$$

The first summation is over the $N$ lattice points of the periodic crystal and the second is over the $N$ points of the first Brillouin zone. The wave-vector $\mathbf{k}(\mathrm{h})$ and the lattice vector $\mathbf{r}(\mathrm{p})$ are defined by:

$$
\begin{aligned}
& \mathbf{r}(p)=p_{\alpha} \mathbf{a}_{\alpha}\left(\alpha=1,2,3 ; \quad p_{\alpha}\right. \text { are integers, sum- } \\
& \text { mation implied }) \\
& \mathbf{k}(h)=2 \pi h_{\alpha} \mathbf{b}_{\alpha}\left(h_{\alpha}=m_{\alpha} / N_{\alpha}, m_{\alpha}=0, \pm 1, \pm 2, \ldots\right)
\end{aligned}
$$

where $\mathbf{a}_{\alpha}$ and $\mathbf{b}_{\alpha}$ are lattice translation vectors and primitive translation vectors of the reciprocal lattice such that $\mathbf{a}_{\alpha} \cdot \mathbf{b}_{\alpha}=\delta_{\alpha \beta}$.

The concentration wave amplitude y $\mathcal{L}\left(\mathbf{k}^{\prime}\right)$ corresponding to the wave vector $\mathbf{k}^{\prime}$ that generates the ordering instability are expressed in terms of normalized (with respect to $c$ ) long range order parameter, $\eta_{\mathrm{n}}$, via the following relation,

$$
\mathcal{L}(\mathbf{k})=\eta_{\mathrm{n}} \cdot c
$$

The normalized order parameter $\left(\eta_{\mathrm{n}}\right)$ is related to the standard order parameter $(\eta)$, as $\eta_{\mathrm{n}}=\eta / \eta_{\max }$, where $\eta_{\max }$ is the maximum order parameter attainable at a given composition. The internal energy, in the pair approximation up to an arbitrary coordination shell, is given as

$$
E=\frac{N}{2} \sum_{h=1}^{N} \mathscr{Z}(\mathbf{k}(h)), \mathcal{L}(\mathbf{k}(h)) \mathcal{L} *(\mathbf{k}(h))
$$

where the star $(*)$ indicates the complex conjugate of the amplitude of the corresponding concentration wave and $\mathscr{Z}(\mathbf{k})$, the Fourier transforms of the pair interactions, are given by $\dagger$

$$
\left.\mathscr{L}(\mathbf{k})=1 / N \sum_{p=1}^{N} \mathscr{J} \mathbf{r}\right) \cdot e^{i \mathbf{k}(h) \cdot \mathbf{r}(p)}
$$

The expression for the configurational entropy is given as

$$
\begin{aligned}
S= & k_{\mathrm{B}} \sum_{\substack{p=1 \\
N}}^{N}[\mathcal{N}(\mathbf{r}(p)) \ln (\mathcal{N}(\mathbf{r}(p)))+(1 \\
& -\mathcal{N}(\mathbf{r}(p))) \ln (1-\mathcal{N}(\mathbf{r}(p)))]
\end{aligned}
$$

which, in terms of sub-lattice probabilities, can be expressed as

$$
S=k_{\mathrm{B}} \sum_{s=1}^{\mathscr{J}} N_{\mathrm{S}}\left[\mathscr{N}_{\mathrm{s}} \ln \mathscr{N}_{\mathrm{s}}+\left(1-\mathcal{N}_{\mathrm{s}}\right) \ln \left(1-\mathcal{N}_{\mathrm{s}}\right)\right]
$$

where $\mathscr{T}$ is the total number of sub-lattices, $\mathcal{N}_{\mathrm{S}}$ and the $\mathscr{N}_{\mathrm{S}}$ are the occupation probability and the number of atoms on the $\mathcal{A}$ h sub-lattice, respectively

The effective pair interactions, $J(\mathbf{k})$ for the wave vector, $\mathbf{k}(\mathrm{h})$ with components $\left(h_{1}, h_{2}, h_{3}\right)$, are related to those in the real space $J_{2}^{(s)}$ as

$$
J(\mathbf{k})=\sum_{(s)} \phi_{\mathbf{k}}^{(s)} J_{2}^{(s)}
$$

where, for the FCC and the BCC lattices, the shell functions, $\phi_{\mathbf{k}}^{(s)}$, for an arbitrary coordination shell " $s$ " are given by the formulae [9]

$$
\begin{aligned}
& \phi_{\mathbf{k}}^{(s)}=\frac{z^{(s)}}{6} \sum_{j=1}^{3} \cos \left(2 \pi h_{1} p_{\mathrm{j}}^{(s)}\right) . \\
& {\left[\cos \left(2 \pi h_{2} p_{\mathrm{j}+1}^{(s)}\right) \cos \left(2 \pi h_{3} p_{\mathrm{j}+2}^{(s)}\right)+\right.} \\
& \left.\cos \left(2 \pi h_{3} p_{\mathrm{j}+1}^{(s)}\right) \cos \left(2 \pi h_{2} p_{\mathrm{j}+2}^{(s)}\right)\right]
\end{aligned}
$$

where $z^{(\mathrm{s})}$ is the number of lattice points in the coordination shell " $s ", p_{\mathrm{j}}^{(s)}$ are integers and half-integers denoting the Cartesian coordinates of a point in the first octant of the shell " $s$ " and " $h$ " denote the Cartesian coordinates in the first Brillouin Zone, as defined already. In Table 2, we have given the calculated values of the shell functions for different $\langle 420\rangle$ wave vectors up to the fourth coordination shell.

The SCW free energy expressions for these FCC-

$\dagger$ The dependences of $\mathbf{r}$ and $\mathbf{k}$ on $p$ and $h$ are assumed if not shown explicitly. 
based ordered super-lattice structures of the (420) family have been derived as follows:

$\mathrm{N}_{4} \mathrm{M}$ (D1 $1_{\mathrm{a}}$ ) structure: The sub-lattice occupation probabilities for the $\mathrm{D} 1_{\mathrm{a}}$ structure are given as

$$
\mathscr{N}_{1}=c+42 \text { and } \mathfrak{N}_{2}=c-2
$$

where 2 is the amplitude of the concentration wave and is related to the normalized (with respect to composition) order parameter $\eta_{\mathrm{n}}$, through the relation, $2=\eta_{\mathrm{n}} \cdot \mathrm{c}$, giving rise to

$$
\mathscr{N}_{1}=c\left(1+4 \eta_{n}\right) \text { and } \mathscr{N}_{2}=c\left(1-\eta_{n}\right)
$$

The internal energy is given by

$$
E_{\mathrm{a}}^{D 1}=\frac{1}{2}\left\{\left(J(000)+\left[\sum_{i=1}^{4} j\left(\frac{i}{5}\langle 420\rangle\right) \eta_{\mathrm{n}}^{2}\right]\right) c^{2}\right\}
$$

and the entropy is given by

$$
S^{D 1_{a}}=-\frac{k_{B}}{5}\left\{4 \mathscr{A}\left(\mathscr{N}_{2}\right)+\mathscr{A}\left(\mathscr{N}_{1}\right)\right\}
$$

with $\mathscr{A} y) \equiv y \ln y+(1-y) \ln (1-y)$.

$\mathrm{N}_{3} \mathrm{M}\left(\mathrm{DO}_{22}\right)$ structure: Here, we have three sublattices with occupation probabilities given as $\mathscr{N}_{1}=c\left(1+\eta_{\mathrm{n}}^{(1)}+2 \eta_{\mathrm{n}}^{(2)}\right), \quad \mathscr{N}_{2}=c\left(1+\eta_{\mathrm{n}}^{(1)}-2 \eta_{\mathrm{n}}^{(2)}\right)$ and $N_{3}=c\left(1-\eta_{\mathrm{n}}^{(1)}\right)$ where $\eta_{\mathrm{n}}^{(1)}$ and $\eta_{n}^{(2)}$ are the order parameters belonging to $\left\langle 1 \frac{1}{2} 0\right\rangle$ and $\langle 100\rangle$ wave vectors, respectively. The internal energy is given by

$$
\begin{aligned}
& E^{D O_{22}}=\frac{1}{2}\{(J(000)+ \\
& {\left[J\left(\frac{1}{4}\langle 420\rangle\right) \eta_{\mathrm{n}}^{(1)^{2}}+J\left(\frac{2}{4}\langle 420\rangle\right) \eta_{\mathrm{n}}^{(2)^{2}}+\right.} \\
& \left.\left.\left.J\left(\frac{3}{4}\langle 420\rangle\right) \eta_{\mathrm{n}}^{(1)^{2}}\right]\right) c^{2}\right\}
\end{aligned}
$$

and the entropy is given by

$$
S^{D O_{22}}=-\frac{k_{B}}{4}\left\{\mathscr{A}\left(N_{1}\right)+\mathscr{A}\left(\mathbb{N}_{2}\right)+2 \mathscr{A}\left(\mathbb{N}_{3}\right)\right\}
$$

$\mathrm{N}_{2} \mathrm{M}\left(\mathrm{Pt}_{2} \mathrm{Mo}\right)$ structure: The sub-lattice occupation probabilities for the $\mathrm{N}_{2} \mathrm{M}$ structure are given as

$$
\mathscr{N}_{1}=c\left(1+2 \eta_{\mathrm{n}}\right) \text { and } \quad \mathscr{N}_{2}=c\left(1-\eta_{\mathrm{n}}\right)
$$

The internal energy is given by

$$
E^{N_{2} M}=\frac{1}{2}\left\{\left(J(000)+\left[\sum_{i=1}^{2} J\left(\frac{i}{3}\langle 420\rangle\right) \eta_{\mathrm{n}}^{2}\right]\right) c^{2}\right\}
$$

and the entropy is given by

$$
\left.S^{N_{2} M}=-\frac{k_{\mathrm{B}}}{3}\left\{2 \mathscr{A}\left(\mathscr{N}_{2}\right)+\mathscr{A} \mathscr{N}_{1}\right)\right\}
$$

\begin{tabular}{|c|c|c|c|c|}
\hline $\begin{array}{l}\text { Coordination shell } \\
\text { (s) } \rightarrow \\
z^{(\mathrm{s})} \rightarrow\end{array}$ & 12 & 2 & 24 & 12 \\
\hline & \multicolumn{4}{|c|}{$p^{(\mathrm{s})}$} \\
\hline $\begin{array}{l}\text { Ordering wave } \\
\text { vector }(\mathbf{k})\end{array}$ & {$\left[\frac{11}{22} 0\right]$} & [100] & {$\left[1 \frac{11}{22}\right]$} & [110] \\
\hline$\frac{1}{3}\langle 420\rangle$ & -3 & 0 & 6 & -3 \\
\hline$\frac{2}{3}\langle 420\rangle$ & -3 & 0 & 6 & -3 \\
\hline$\frac{1}{5}\langle 420\rangle$ & -3 & 1 & 4 & -3 \\
\hline$\frac{2}{5}\langle 420\rangle$ & -3 & 1 & 4 & -3 \\
\hline$\frac{3}{5}\langle 420\rangle$ & -3 & 1 & 4 & -3 \\
\hline$\frac{4}{5}\langle 420\rangle$ & -3 & 1 & 4 & -3 \\
\hline$\frac{1}{4}\langle 420\rangle$ & -4 & 2 & 8 & -4 \\
\hline$\frac{2}{4}\langle 420\rangle$ & -4 & 6 & -8 & 12 \\
\hline$\frac{3}{4}\langle 420\rangle$ & -4 & 2 & 8 & -4 \\
\hline
\end{tabular}

$\mathrm{N}_{2} \mathrm{M}_{2}$ (I4/amd) structure: The sub-lattice occupation probabilities for the $\mathrm{N}_{2} \mathrm{M}_{2}$ structure are given as

$$
\mathscr{N}_{1}=c\left(1+\eta_{\mathrm{n}}\right) \text { and } \quad \mathscr{N}_{2}=c\left(1-\eta_{\mathrm{n}}\right)
$$

The internal energy is given by

Table 2. The shell functions $\phi_{\mathbf{k}}^{(s)}$ 's calculated upto the 4th coordination shell for fcc lattice for different wave vectors $\mathbf{k}$. $z^{(\mathrm{s})}$ represent the coordination number for the $s$ th shell and $p^{(\mathrm{s})}$ denotes the cartesian coordinates of a point in the first octant of the shell

$$
\begin{aligned}
& E^{N_{2} M_{2}}=\frac{1}{2} \\
& \left\{\left(J(000)+\left[J\left(\frac{1}{4}\langle 420\rangle\right)+J\left(\frac{3}{4}\langle 420\rangle\right)\right] \eta_{\mathrm{n}}^{2}\right) c^{2}\right\}
\end{aligned}
$$

and the entropy is given by

$$
\left.S^{N_{2} M_{2}}=-\frac{k_{B}}{2}\left\{\mathscr{A}\left(N_{2}\right)+\mathscr{A} \mathscr{N}_{1}\right)\right\}
$$

4.2.1. Ground state analysis. At zero temperature, there is no contribution from entropy to the free energy of a solid solution and therefore its stability is governed by the internal energy alone, which is a function of effective pair interactions. In Table 1, we have given the values of these EPIs $\left(J_{2}^{(i)}(c), i=1,4\right)$ 
up to the fourth nearest neighbor pairs for FCC-based Ni-Mo alloys, calculated using the ASR-OP method.

The occurrence of ordering and clustering instabilities in solid solutions has been analyzed by Clapp and Moss [25] and de Fontaine [9, 12], whereas the interplay between the clustering and ordering instabilities have been examined by Kulkarni et al. [4], in terms of concentration waves with wave vector $\mathbf{k}$ which terminate at the so-called special points in the reciprocal lattice.

These special points are of interest as these are the locations of the extrema of the k-space potential energy function, $J(\mathbf{k})$, i.e. corresponding to $\partial J(\mathbf{k}) / \partial \mathbf{k}=0$. The nature of the extrema (minima/maxima/saddle point) is determined by the second derivative of the free energy function. Therefore, concentration waves with a wave vector $\mathbf{k}$ which corresponds to a minimum of $J(\mathbf{k})$ are characterized by maximum value of instability temperature $\left(T_{\mathrm{i}}\right)$ and the amplification rate $(\alpha(\mathbf{k}))$. A FCC solid solution may exhibit the following special point instabilities (a) $\langle 000\rangle$ or the clustering instability leading to spinodal phase separation and (b) $\langle 100\rangle,\left\langle\frac{1}{2} \frac{1}{2} \frac{1}{2}\right\rangle$ and $\left\langle 1 \frac{1}{2} 0\right\rangle$ ordering instabilities. The ordered structures generated by the amplification of a single variant of the $\left\langle\frac{1}{2} \frac{1}{2} \frac{1}{2}\right\rangle$ or the $\left\langle 1 \frac{1}{2} 0\right\rangle$ waves obey the Landau-Lifshitz rules I, II and III [9, 12] and are, therefore, candidates for the order-disorder transformation of the second kind. In the case of $\left\langle\frac{1}{2} \frac{1}{2} \frac{1}{2}\right\rangle$ and $\left\langle 1 \frac{1}{2} 0\right\rangle$ ordering the coefficients of the third order term in the Landau-Lifshitz free energy expansion is equal to zero from symmetry considerations.

In Table 3, we have given the values of the EPIs in the reciprocal space calculated using equation (25), for various wave vectors, $\mathbf{k}$, corresponding to all the members of (420) family. Kulkarni et al. [4] have analyzed the $\langle 100\rangle,\left\langle\frac{1}{2} \frac{1}{2} \frac{1}{2}\right\rangle$ and $\left\langle 1 \frac{1}{2} 0\right\rangle$ special point ordering instabilities by considering (concentrationindependent) effective pair interactions up to the third nearest neighbor pairs using the mean-field based SCW model. Their results for the $\left\langle 1 \frac{1}{2} 0\right\rangle$ special point ordering instability in the $J_{2}^{(2)} / J_{2}^{(1)}-J_{2}^{(3)} / J_{2}^{(1)}$ space are shown in Fig. 5 where the horizontally-hatched portion represents the region of absolute minimum of $J\left(1 \frac{1}{2} 0\right)$ in the EPI space. The region below the dashed line represents region of phase separation of ordered solid solution into a pure $\mathrm{A}$ and an ordered $A_{1-\mathrm{c}} B_{\mathrm{c}}$ phase and the vertically-hatched portion represents region of combined $\left\langle 1 \frac{1}{2} 0\right\rangle$ ordering and subsequent phase separation. We have calculated these EPIs (concentration dependent) up to the fourth coordination shell for the FCC Ni-Mo alloys using the ASROP method. Our values of $J_{2}^{(2)} / J_{2}^{(1)}$ and $J_{2}^{(3)} / J_{2}^{(1)}$ at the four compositions viz. 20, 25, 33 and 50 at\% of Mo have been given in Table 1 and have also been superimposed onto Fig. 5. These ratios of our calculated EPIs have been observed to be in the region corresponding to the minimum of $J\left(1 \frac{1}{2} 0\right)$ i.e. $\left\langle 1 \frac{1}{2} 0\right\rangle$ ordering instability exists in the Ni-Mo alloys.
Table 3. The Fourier transforms of the EPIs calculated using the ASROP method, for various wave vectors, $\mathbf{k}$, corresponding to all the members of (420) series

\begin{tabular}{|c|c|c|c|}
\hline $\begin{array}{l}\text { Composition } \\
\text { (at\% Mo) }\end{array}$ & $J(000)(\mathrm{mRy})$ & $\mathbf{k}$ & $J(\mathbf{k})(\mathrm{mRy})$ \\
\hline \multirow[t]{4}{*}{20} & 42.966 & $\frac{1}{5}\langle 420\rangle$ & -11.357 \\
\hline & & $\frac{2}{5}\langle 420\rangle$ & -11.357 \\
\hline & & $\frac{3}{5}\langle 420\rangle$ & -11.357 \\
\hline & & $\frac{4}{5}\langle 420\rangle$ & -11.357 \\
\hline \multirow[t]{3}{*}{25} & 28.298 & $\frac{1}{4}\langle 420\rangle$ & -11.749 \\
\hline & & $\frac{2}{4}\langle 420\rangle$ & -6.643 \\
\hline & & $\frac{3}{4}\langle 420\rangle$ & -11.749 \\
\hline \multirow[t]{2}{*}{33} & 9.002 & $\frac{1}{3}\langle 420\rangle$ & -3.609 \\
\hline & & $\frac{2}{3}\langle 420\rangle$ & -3.609 \\
\hline \multirow[t]{2}{*}{50} & 1.795 & $\frac{1}{4}\langle 420\rangle$ & -3.899 \\
\hline & & $\frac{3}{4}\langle 420\rangle$ & -3.899 \\
\hline
\end{tabular}

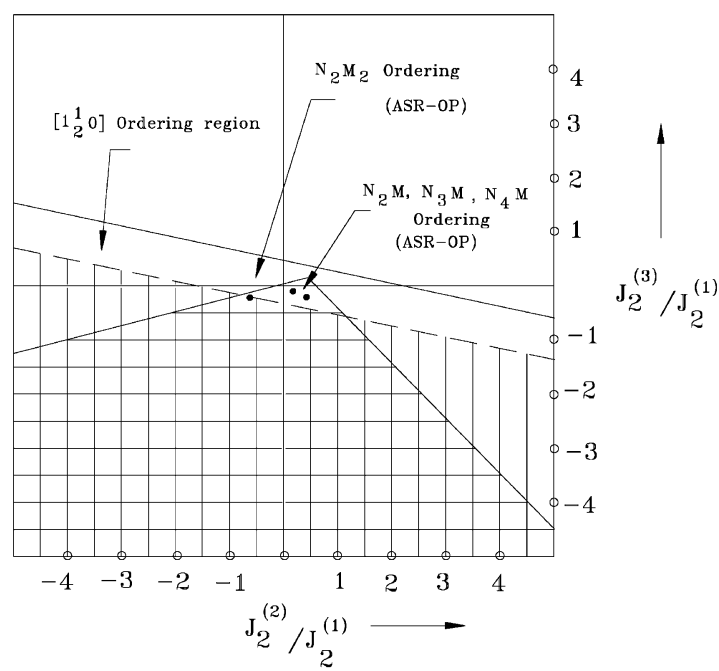

Fig. 5. The $\left\langle 1 \frac{1}{2} 0\right\rangle$ special point instability diagram, calculated using the SCW model, marking different regions in the EPIspace where clustering and/or ordering instabilities exist. Superimposed on it are our ASR-OP calculated ratios of EPIs.

The ordering energies $\left(E_{\Phi}^{\text {ord }}=E_{\Phi}-E^{\text {disordered }}\right)$ of all the four ordered structures $(\Phi)$ which are members of (420) family, calculated using the SCW model [10, 24], at different compositions are given in Table 4. We will now examine the relative ground state phase 
Table 4. The SCW ordering energy ( $\left.E_{\Phi}^{\text {ord }}=E_{\Phi}-E^{\text {disordered }}\right)$ values of all the ordered phases $(\Phi)$ of (420) family of Ni-Mo alloy system as a function of composition.

\begin{tabular}{lcr}
\hline Phase $(\Phi)$ & $\begin{array}{c}\text { Composition }(\text { at } \% \\
\text { Mo) }\end{array}$ & $E_{\Phi}^{\text {ord }}(\mathrm{K})$ \\
\hline \multirow{2}{*}{$\mathrm{N}_{4} \mathrm{M}$} & 0.20 & -143.45 \\
& 0.25 & -224.15 \\
$\mathrm{~N}_{3} \mathrm{M}$ & 0.20 & -95.18 \\
& 0.25 & -148.71 \\
$\mathrm{~N}_{2} \mathrm{M}$ & 0.20 & -22.79 \\
& 0.25 & -35.61 \\
& 0.33 & -62.05 \\
$\mathrm{~N}_{2} \mathrm{M}_{2}$ & 0.20 & -24.62 \\
& 0.25 & -38.47 \\
& 0.50 & -153.89 \\
\hline
\end{tabular}

stability of these ordered superstructures $(\Phi)$ from their ordering energy values, as calculated above, and compare our results with those observed experimentally. For $\mathrm{Ni}_{3} \mathrm{Mo}$ based alloys, we immediately see that

$$
E_{\mathrm{N}_{2} \mathrm{M}_{2}}^{\text {ord }}<E^{\text {disordered }} \text { at } c_{\mathrm{Mo}}=0.25
$$

indicating that $\mathrm{N}_{2} \mathrm{M}_{2}$ ordering instability exists in the disordered solid solution.

Since the introduction of a $\langle 100\rangle$ concentration wave (secondary ordering) in a $\left\langle 1 \frac{1}{2} 0\right\rangle$ ordered alloy of $\mathrm{N}_{3} \mathrm{M}$ composition gives rise to $\mathrm{DO}_{22}$ structure, the $\mathrm{DO}_{22}$ structure should be more stable than the offstoichiometric $\mathrm{N}_{2} \mathrm{M}_{2}$ structure. From Table 4, we see that

$$
E_{\mathrm{N}_{3} \mathrm{M}}^{\text {ord }}<E_{N_{2} M_{2}}^{\text {ord }} \text { at } c_{\mathrm{Mo}}=0.25 \text {. }
$$

The secondary $\left\langle 1 \frac{1}{2} 0\right\rangle$ ordering in a direction perpendicular to the original $\left\langle 1 \frac{1}{2} 0\right\rangle$ vector gives rise to a structure which contains an ordered arrangement of $\mathrm{N}_{4} \mathrm{M}$ and $\mathrm{N}_{2} \mathrm{M}$ subunit cell clusters. The condition for the stability of such a structure relative to the $\mathrm{N}_{2} \mathrm{M}_{2}$ structure can be seen to be satisfied from our ASROP calculations as

$$
\frac{1}{2}\left[E_{\mathrm{N}_{4} \mathrm{M}}^{\text {ord }}+E_{\mathrm{N}_{2} \mathrm{M}}^{\text {ord }}\right]<E_{\mathrm{N}_{2} \mathrm{M}_{2}}^{\text {ord }} \text { at } c_{\mathrm{Mo}}=0.25 .
$$

Hence, the super-imposition of (a) an $\langle 100\rangle$ and an $\left\langle 1 \frac{1}{2} 0\right\rangle$ concentration waves or (b) two mutually perpendicular $\left\langle 1 \frac{1}{2} 0\right\rangle$ concentration waves always results in a structure of lower internal energy than the offstoichiometric $\mathrm{N}_{2} \mathrm{M}_{2}$ structure. Further, we also see that

$$
E_{\mathrm{N}_{3} \mathrm{M}}^{\text {ord }}<\frac{1}{2}\left[E_{\mathrm{N}_{4} \mathrm{M}}^{\text {ord }}+E_{\mathrm{N}_{2} \mathrm{M}}^{\text {ord }}\right] \text { at } c_{\mathrm{Mo}}=0.25
$$

This prediction of stability of $\mathrm{DO}_{22}$ structure compared to the mixture of $\mathrm{D} 1_{\mathrm{a}}$ and $\mathrm{Pt}_{2} \mathrm{Mo}$ is contrary to experimental findings. We, however, notice that the difference between the formation energies of $\mathrm{N}_{3} \mathrm{M}$ on one hand and a mixture of $\mathrm{N}_{4} \mathrm{M}$ and $\mathrm{N}_{2} \mathrm{M}$ on the other, is extremely small. Since these two alternatives are energetically comparable, the preference for one of them to form changes with minor variation in alloy composition. While in binary alloys close to $\mathrm{Ni}_{75} \mathrm{Mo}_{25}$ the $\mathrm{N}_{4} \mathrm{M}+\mathrm{N}_{2} \mathrm{M}$ develops during the early stage of ordering, the $\mathrm{N}_{3} \mathrm{M}\left(\mathrm{DO}_{22}\right)$ should become more favorable in several ternary alloys. It may also be emphasized that this simple version of the ASR-OP used here does not take into account local lattice distortions because of large size difference between the $\mathrm{Ni}$ and Mo atoms. The local strain contributes towards the internal energy and may have considerable effect on the rather small EPIs. The ASR, in an extended form, is capable of taking into account the effect of local strains in the internal energy [27].

The ground state stability analysis at $c_{\mathrm{Mo}}=0.20$ shows the hierarchy as

$$
E_{\mathrm{N}_{4} \mathrm{M}}^{\text {ord }}<E_{\mathrm{N}_{2} \mathrm{M}_{2}}^{\text {ord }}<E^{\text {disordered }}
$$

which is consistent with the experimental findings.

4.2.2. Finite temperature analysis. We have calculated free energies of these superstructures, as functions of temperature, composition and order parameter using the static concentration wave model [24] where we have taken the single-site approximation for the estimation of entropy. Under this approximation, the long-range correlation are properly treated but short-range correlations beyond a single site are ignored.

The instability temperature $\left(T_{\mathrm{i}}^{-}\right)$, defined as the temperature corresponding to the onset of ordering instability in the solid solution, is given in the SCW model by

$$
T_{\mathrm{i}}^{-}=-\frac{J(k)}{k_{\mathrm{B}}} c(1-c)
$$

for the ordering wave vector $\mathbf{k}$.

The ordering free energy $\left(F^{\text {ord }}=F^{\Phi}-F^{\text {disord }}\right)$ as a function of normalized order parameter $\left(\eta_{\mathrm{n}}\right)$ for the $\mathrm{N}_{2} \mathrm{M}_{2}$ phase at the stoichiometric composition $c_{\mathrm{Mo}}=0.50$, and at $c_{\mathrm{Mo}}=0.20$, are plotted in Fig. 6 . The temperature range has been chosen to be around the instability temperature $\left(T_{\mathrm{i}} \equiv T_{\mathrm{c}}\right)$ at a given composition. These Landau plots, which we have generated from our first-principles calculations, show that the curvature of the $F^{\text {ord }}$ vs $\eta$ plot changes the sign from positive to negative at $T_{\mathrm{c}}$, as expected from a second order phase transition. The $\mathrm{N}_{2} \mathrm{M}_{2}$ instability temperatures for different alloy compositions, in the binary Ni-Mo system, essentially give the ordering spinodal line which was reported (schematically) earlier by de Fontaine [9].

Figure 7 shows the variation of ordering free 


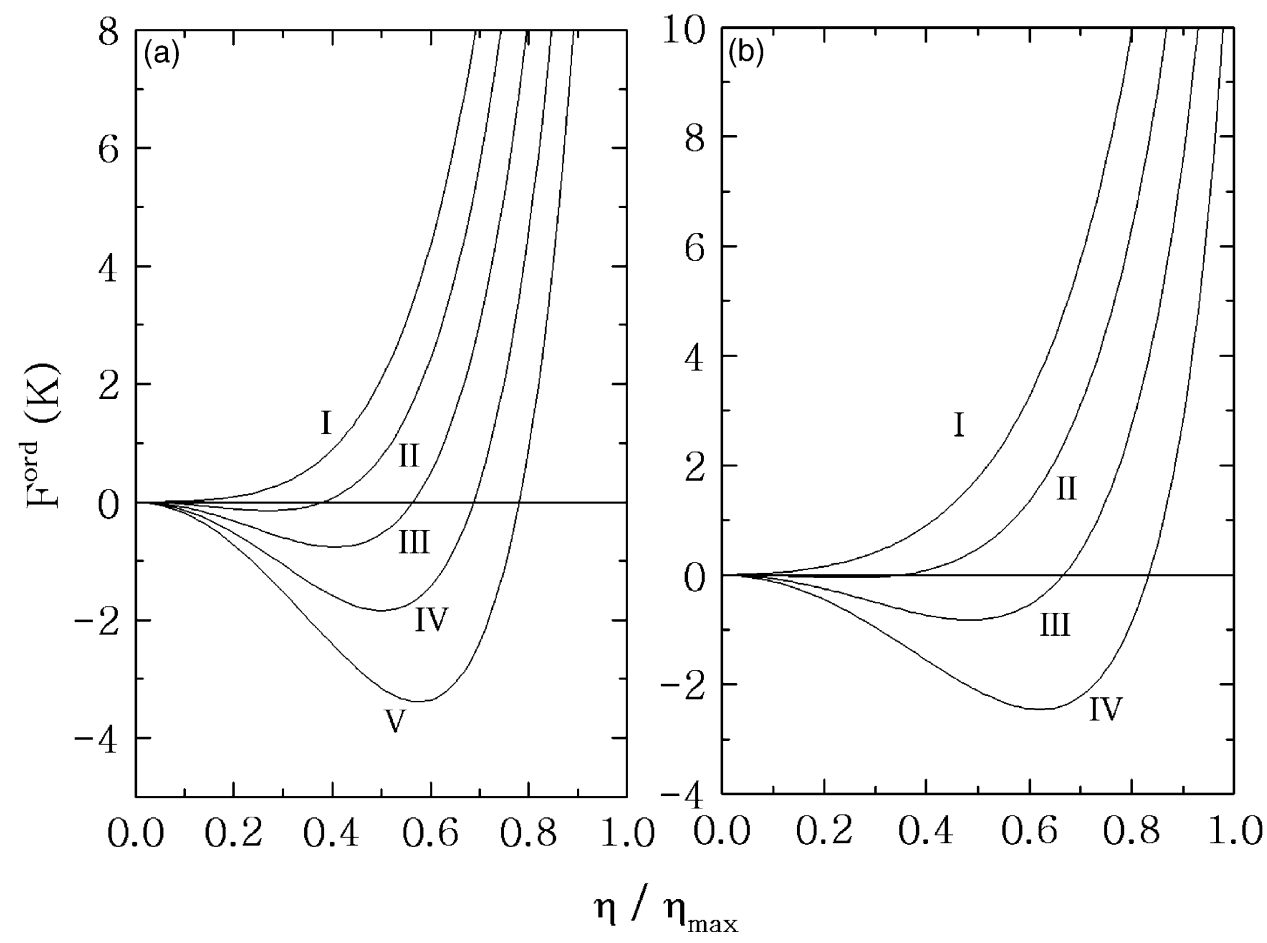

Fig. 6. The ordering free energy of the $\mathrm{N}_{2} \mathrm{M}_{2}$ phase plotted as a function of $\eta_{\mathrm{n}}$ at the stoichiometric composition, (a) $c_{\mathrm{Mo}}=0.50$ and at (b) $c_{\mathrm{Mo}}=0.20$, respectively, at several temperatures around the instability temperature ( $T_{\mathrm{i}} \equiv T_{\mathrm{c}}$ ). (a) Plots I-V correspond to $\left[T /\left(T_{\mathrm{c}}\right)\right]=1.02,0.98,0.95$ and 0.92 , respectively. (b) Plots I-IV correspond to $\left[T /\left(T_{\mathrm{c}}\right)\right]=1.01,0.95,0.88$ and 0.83 , respectively.

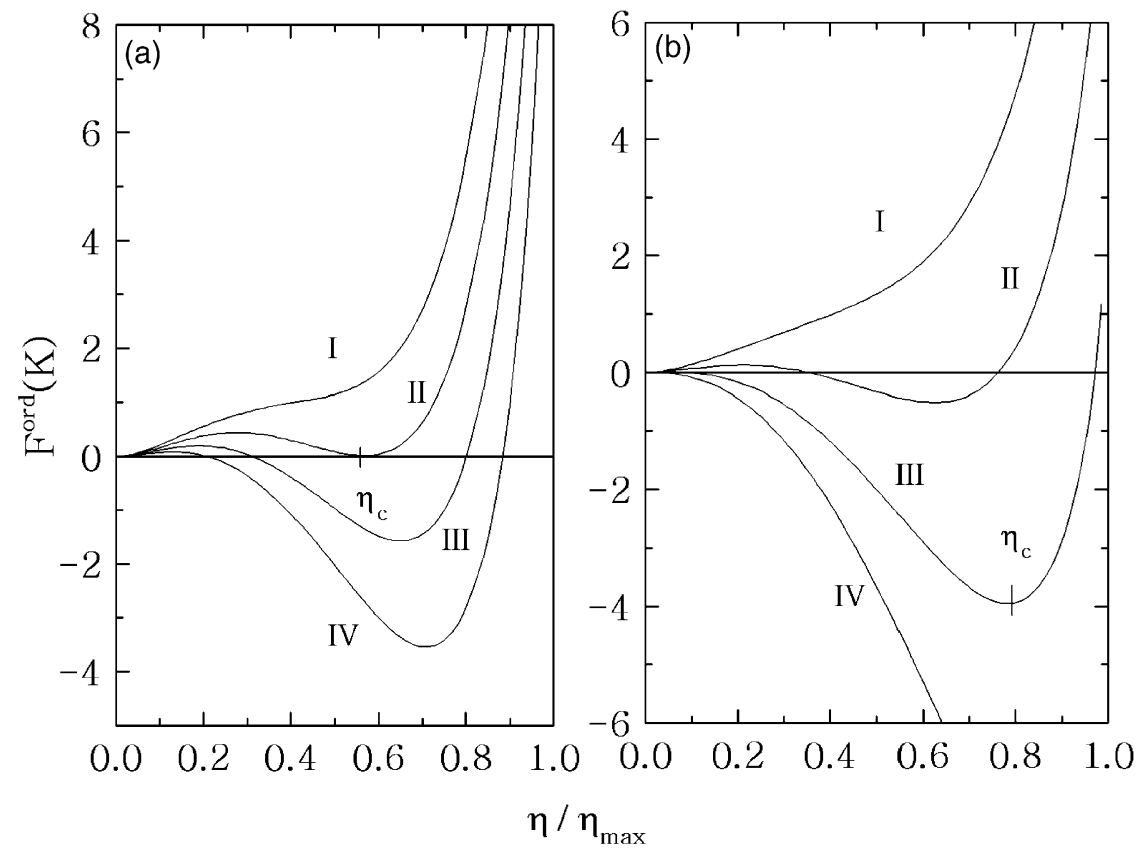

Fig. 7. The variation of ordering free energy of $\mathrm{N}_{4} \mathrm{M}$ phase with the order parameter at the stoichiometric composition (a) $c_{\mathrm{Mo}}=0.2$. and at (b) $c_{\mathrm{Mo}}=0.25$ respectively, at several temperatures around the transition temperature $\left(T_{\mathrm{c}}\right)$. (a) Plots I-IV correspond to $\left[T /\left(T_{\mathrm{c}}\right)\right]=1.03,1.00,0.97$ and 0.94 , respectively. (b) Plots IIV correspond to $\left[T /\left(T_{\mathrm{c}}\right)\right]=1.04,0.97,0.90$ and 0.83 , respectively. 
energy of $\mathrm{D} 1_{\mathrm{a}}$ structured $\mathrm{N}_{4} \mathrm{M}$ phase with the order parameter at the stoichiometric composition $c_{\mathrm{Mo}}=0.2$ and at $c_{\mathrm{Mo}}=0.25$. It may be noted that the plot (II) in Fig. 7(a) corresponds to the order-disorder transition temperature, $T_{\mathrm{c}}$, at which the disordered state $(\eta=0)$ and the ordered state $\left(\eta=\eta_{\mathrm{c}}\right)$ have the same free energy. The hump between these two states represents free energy barrier at $T_{\mathrm{c}}$, which is characteristic of first order transitions. Figure 7(b) illustrates that at a certain degree of under-cooling, instability with respect to ordering develops, as reflected by the negative curvature of the $F^{\text {ord }}$ versus $\eta$ plots at $\eta=0$. The temperature at which such an instability develops is defined as the instability temperature $\left(T_{\mathrm{i}}\right)$ which can be determined from these plots for different super-lattice structures (both equilibrium and meta-stable) belonging to the (420) family.

The $\mathrm{DO}_{22}$-structured $\mathrm{N}_{3} \mathrm{M}$ phase, which closely competes with the $\mathrm{HCP}$-based $\mathrm{DO}_{\mathrm{a}}$ structure, has two order parameters $\left(\eta_{\mathrm{n}}^{(1)}\right.$ and $\left.\eta_{n}^{(2)}\right)$ belonging to $\left\langle 1 \frac{1}{2} 0\right\rangle$ and $\langle 100\rangle$ wave vectors, respectively. The free energies of ordering of the $\mathrm{DO}_{22}$ phase as a function of these two order parameters at the composition $c_{\mathrm{MO}}=0.25$ are shown in Fig. 8. The stability domain of the $\mathrm{DO}_{22}$ phase corresponds to the region of negative free energy values.

Let us now consider a situation when a first order ordering process competes with a second order ordering. The completely disordered $\mathrm{Ni}_{4} \mathrm{Mo}$ $\left(c_{\mathrm{MO}}=0.20\right)$ alloy experiences both kinds of ordering tendencies viz. the second order $\left\langle 1 \frac{1}{2} 0\right\rangle$ ordering and the first order $\frac{1}{5}\langle 420\rangle$ ordering. For illustrative purpose, we have calculated the free energy of this alloy $F^{N i_{4} M o}\left(\eta_{\mathrm{n}}^{\left\langle 1 \frac{1}{2} 0\right\rangle}, \eta_{n}^{\frac{1}{5}\langle 420\rangle}\right)$ at a given temperature as

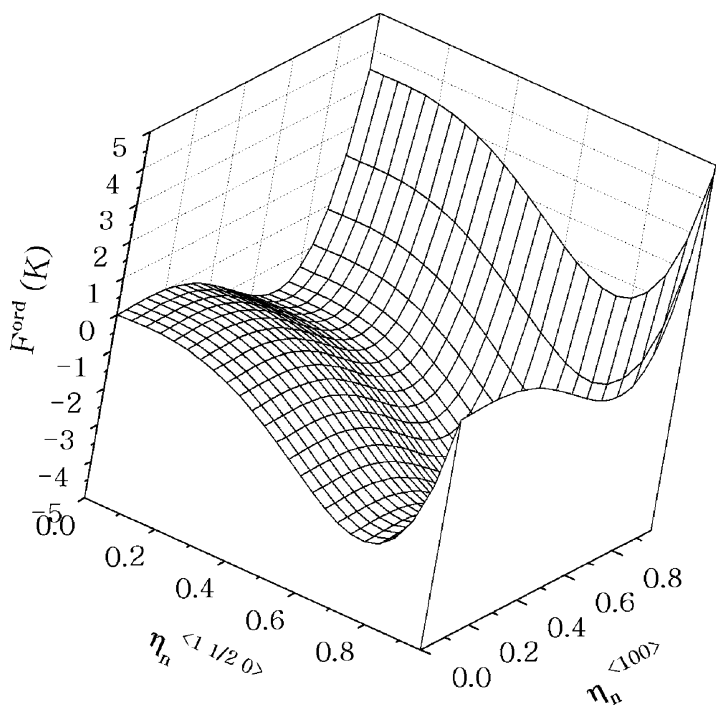

Fig. 8. The variation of free energy of ordering of $\mathrm{DO}_{22}$ phase with the order parameters $\left(\eta_{\mathrm{n}}\left\langle 1 \frac{1}{2} 0\right\rangle\right.$ and $\left.\eta_{\mathrm{n}}\langle 100\rangle\right)$ corresponding to $\left\langle 1 \frac{1}{2} 0\right\rangle$ and $\langle 100\rangle$ wave vectors at $\left[T /\left(T_{\mathrm{c}}\right)\right]=0.92$.

$$
\begin{aligned}
& F^{N i}{ }_{4}^{M o}\left(\eta_{\mathrm{n}}^{\left\langle 1 \frac{1}{2} 0\right\rangle}, \eta_{\mathrm{n}}^{\frac{1}{5}\langle 420\rangle}\right)= \\
& \frac{1}{2}\left[F^{N_{4} M}\left(\eta_{\mathrm{n}}^{\frac{1}{5}\langle 420\rangle}\right)+F^{N_{2} M_{2}}\left(\eta_{\mathrm{n}}^{\left\langle 1 \frac{1}{2} 0\right\rangle}\right)\right]
\end{aligned}
$$

The criterion of stability with respect to fluctuation in order parameter for a given wave vector can be determined by examining the curvature of the $F$ vs. $\eta$ plots at $\eta=0$. Our results have been given in Fig. 9, for illustrating the following 4 distinct situations:

1. Positive curvatures for both $\left\langle 1 \frac{1}{2} 0\right\rangle$ and $\frac{1}{5}\langle 420\rangle$ ordering, implying stability of the disordered state (Fig. 9(a))

2. Negative curvature for $\left\langle 1 \frac{1}{2} 0\right\rangle$ and positive curvature for $\frac{1}{5}\langle 420\rangle$, implying instability of the system for $\left\langle 1 \frac{1}{2} 0\right\rangle$ ordering, and no ordering tendency along $\frac{1}{5}\langle 420\rangle$ (Fig. 9(b))

3. Negative curvature for $\left\langle 1 \frac{1}{2} 0\right\rangle$, and positive for $\frac{1}{5}\langle 420\rangle$ at $\eta=0$, but a dip in the free energy plot with respect to $\frac{1}{5}\langle 420\rangle$ near $\eta=0.8$. This implies that the system experiences simultaneous ordering tendencies towards $\left\langle 1 \frac{1}{2} 0\right\rangle$ ordering (second order) and $\frac{1}{5}\langle 420\rangle$ (first order) (Fig. 9(c))

4. Negative curvature along both $\left\langle 1 \frac{1}{2} 0\right\rangle$ and $\frac{1}{5}\langle 420\rangle$, i.e. system experiences instabilities for $\left\langle 1 \frac{1}{2} 0\right\rangle$ and $\frac{1}{5}\langle 420\rangle$ ordering simultaneously (Fig. 9(d)).

Although $\mathrm{D} 1_{\mathrm{a}}$ is the stable equilibrium structure at $c_{\mathrm{Mo}}=0.20$, a stronger tendency for the development of $\left\langle 1 \frac{1}{2} 0\right\rangle$ ordering can be noticed in the initial stages of ordering, as reflected by a larger negative curvature of the free energy surface at $\eta\left\langle 1 \frac{1}{2} 0\right\rangle=\eta \frac{1}{5}\langle 420\rangle=0$ along the $\eta\left\langle 1 \frac{1}{2} 0\right\rangle$ axis compared to that along with that along $\eta \frac{1}{5}\langle 420\rangle$ axis (Fig. 9(a)).

The curvature of the free energy surface is negative in both the directions in Fig. 9(d) which suggest that homogeneous ordering is possible for both the ordering processes. A mixed state consisting of concentration waves with wave vector ranging from $\left\langle 1 \frac{1}{2} 0\right\rangle$ to $\frac{1}{5}\langle 420\rangle$ is encountered on the path of the ordering process at sufficiently low temperatures [26].

Although this first-principles approach has predicted the general trends of the complex ordering processes encountered in this system, precise determination of the transition temperatures corresponding to different superlattice structures would require further refinements.

\section{CONCLUSION}

From the above analysis, it is clear that the transformation behavior of ordering in alloys in terms of ordering instabilities can been studied using first-principles TB-LMTO method coupled with ASR-OP technique. This method in conjunction with the meanfield statistical mechanics based SCW model has been used to determine the free energies of ordered phases as functions of temperature, composition and order 
(a)

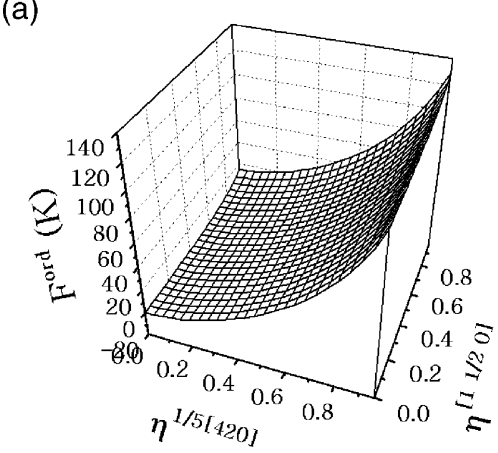

(c)

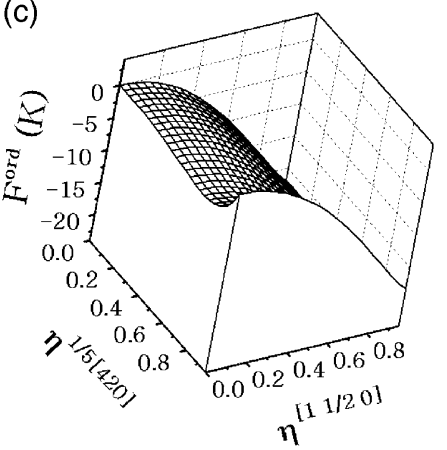

(b)
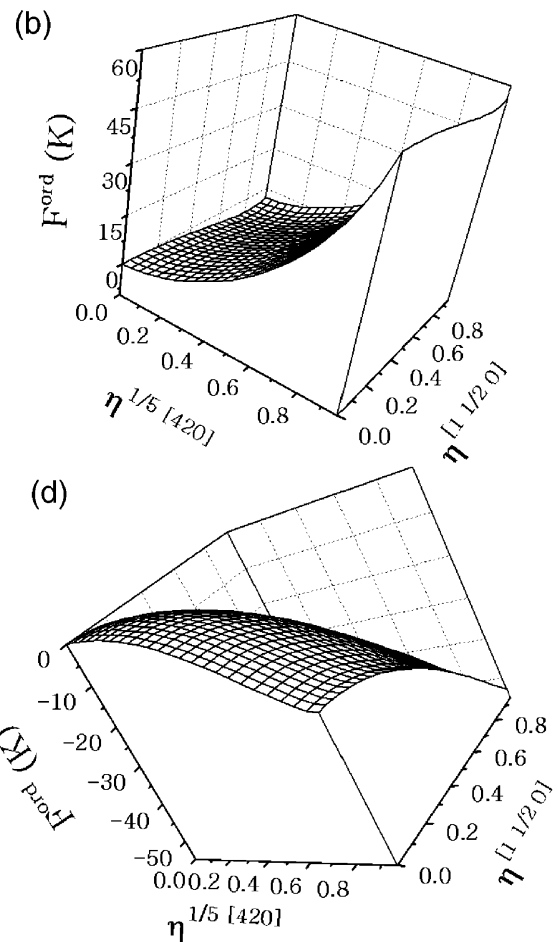

Fig. 9. The ordering free energy of the $\mathrm{Ni}_{4}$ Mo-based alloy, exhibiting the $\left\langle 1 \frac{1}{2} 0\right\rangle$ and the $\frac{1}{2}\langle 420\rangle$ ordering tendencies, plotted as a function of order parameters for the corresponding ordering wave vector at four different temperatures, (a) to (d) in decreasing temperature sequence (see text for details).

parameter. The SCW model gives the correct ground state stability sequence as observed experimentally. Furthermore, it has been shown that such an approach can be used to study the complex transformation behavior, involving several competing superstructures. This approach has been found to be useful in examining situations in which first and second order processes compete, as in case of the present Ni-Mo system.

\section{REFERENCES}

1. Shunk, F. A., Constitution of Binary Alloys, Second Suppl. McGraw-Hill, New York, 1969.

2. Das, S. K. and Thomas, G., Physica Status Solidi (a), 1974, 21, 177.

3. Brooks, C. R., Spruiell, J. E. and Stansbury, E. E., International Metal Reviews, 1984, 29(3), 210.

4. Kulkarni, U. D. and Banerjee, S., Acta Metall., 1988, 36(4), 413.

5. Banerjee, S. and Urban, K., Phys. Stat. Sol. (a), 1984, 81, 145.

6. Banerjee, S., Kulkarni, U. D. and Urban, K., Acta Metall., 1989, 37(1), 35.

7. Das, S. K., Okomoto, P. R., Fischer, P. M. J. and Thomas, G., Acta Metall., 1973, 21, 913.

8. Van Tendeloo, G., Amelinckx, D. and de Fontaine, D., Acta Crystallogr., 1985, B41, 281.

9. de Fontaine, D., Acta Metall., 1975, 23, 553.

10. Khachaturyan, A. G., Prog. Mater. Sci., 1978, 22, 1.

11. Okamoto, P. R. and Thomas, G., Acta Metall., 1971, 19, 825

12. de Fontaine, D., in Solid-State Phase Transformations, eds.
H. I. Aaronson, D. E. Laughlin, R. F. Sekerka and C. M. Wayman. Metall. Soc. A.I.M.E, Warrendale, PA, 1981, p. 25.

13. Arya, A., Das, G. P., Banerjee, S. and Patni, M. J., J. Phys.: Condens Matter, 1998, 38, 8459.

14. Saha-Dasgupta, T. and Mookerjee, A., J. Phys.: Condens Matter, 1997, 9, 2179.

15. Haydock, R., Solid State Physics, Vol. 35. Academic Press, New York, 1980, p. 216.

16. Haydock, R., in Methods of Electronic Structure Calculations, eds. O. K. Andersen, V. Kumar and A. Mookerjee. World Scientific, Singapore, 1994, p. 125.

17. Mookerjee, A., in Methods of Electronic Structure Calculations, eds. O. K. Andersen, V. Kumar and A. Mookerjee. World Scientific, Singapore, 1994, p. 193.

18. Luchini, M. U. and Nex, C. M. M., J. Phys. Conds. Matter, 1987, 20, 3125.

19. Saha, T., Dasgupta, I. and Mookerjee, A., J. Phys.: Condens Matter, 1994, 6, L245; Saha, T., Dasgupta I. and Mookerjee, A., Phys. Rev., 1994, B50, 13267.

20. Andersen, O. K. and Jepsen, O., Phys. Rev. Lett., 1984, 53, 2571.

21. Andersen, O. K., Jepsen, O. and Glötzel, D., in Highlights in Condensed Matter Theory, eds. F. Bassani, F. Fumi and M. P. Tosi. North-Holland, New York, 1985, p. 59.

22. Burke, N. R., Surf. Sci., 1976, 58, 349.

23. Dasgupta, I., Saha, T. and Mookerjee, A., Phys. Rev., 1995, B51, 3413.

24. Khachaturyan, A. G., Theory Of Structural Transformations In Solids. Wiley, New York, 1983.

25. Clapp, P. C. and Moss, S. C., Phys. Rev., 1966, 142, 418.

26. Banerjee, S., Urban, K. and Wilkens, M., Acta Metall., 1984, 32, 299.

27. Saha, T. and Mookerjee, A., J. Phys.: Condens. Matt., 1996, 8, 2915. 